\title{
THE EMISSIONS OF BROADBAND ELECTROSTATIC NOISE IN THE NEAR VICINITY OF THE SHUTTLE ORBITER
}

\author{
K. S. HWANG \\ NRC, Space Science Laboratory. NASA Marshall Space Flight Center, Huntsville, AL 35812, U.S.A. \\ N. H. STONE \\ Space Science Laboratory, NASA Marshall Space Flight Center, Huntsville, AL 35812, U.S.A. \\ K. H. WRIGHT, Jr. \\ Physics Department, The University of Alabama in Huntsville, Huntsville, AL 35899, U.S.A \\ and \\ U. SAMIR \\ Tel-Aviv University, Ramat-Aviv, Israel, and Space Physics Research Laboratory, \\ The University of Michigan, Ann Arbor, MI 48109, U.S.A.
}

\section{(Received in final form 16 April 1987)}

\begin{abstract}
Measurements of the Space Shuttle environment from the STS-3 and Spacelab 2 missions indicate the presence of oblique ion streams and broadband electrostatic noise. A two-dimensional theoretical model is applied to study a possible causal relationship between the ion streams and the broadband noise; especially in terms of the ion acoustic wave and ion-ion wave modes. This model predicts the generation of waves with frequencics ranging from the ion cyclotron frequency up to valucs grcater than the ion plasma frequency, with the maximum growth rate occurring in the $10 \mathrm{kHz}$ range. These results are consistent with the observational data from the $S T S-3$ mission. The model also shows that these two wave modes can co-exist only when the wave vectors of the two wave modes are nearly perpendicular. The parametric dependence of the wave instabilities on the plasma parameters, e.g. $N_{\mathrm{b}} / N_{\mathrm{e}}, T_{\mathrm{e}} / T_{\mathrm{i}}$ and the inclination of the wave propagation vector, is also studied.
\end{abstract}

\section{INTRODUCTION}

Wave-particle interactions resulting from the acceleration of ions in a collisionless plasma are of fundamental significance in space plasma physics. Previous observations from the IMP 7 satellite (Scarf et al., 1974) and the IMP 8 satellite (Gurnett et al., 1976) indicate that field-aligned streams are associated with a continuum of electrostatic ion waves, ranging from $10 \mathrm{~Hz}$ up to several kilohertz in the Earth's magnetospheric region. Recent theoretical treatments of ion stream-background plasma interactions (Grabbe and Eastman, 1984; Omidi, 1985; Akimoto and Omidi, 1986; Ashour-Abdalla and Okuda, 1986) also predict the occurrence of broadband electrostatic noise with wave vectors in every direction and with the most intense wave occurring in a direction nearly perpendicular to the field-aligned current. This behavior is consistent with the IMP 8 observations (Gurnett et al., 1976).

More recently, measurements from the STS-3 and Spacelab 2 Space Shuttle missions have also revealed the existence of high inclination secondary ion streams in the Orbiter's near environment (Stone et al., 1983,
1986). The current density of these ion streams can be a significant fraction of the ram current density, ${ }^{*}$ and it is thought that these ion streams may be associated with broadband electrostatic noise, ranging from $30 \mathrm{~Hz}$ up to $178 \mathrm{kHz}$, that was observed on the same missions (Shawhan et al., 1984; Murphy et al., 1983). In addition, electron-ambient ion temperature ratios as high as 6 were reported by Raitt ct al., (1984) and Siskind et al., (1984) based on electron and ion measurements made near the Orbiter during the STS3 mission. The electrons may have been heated by wave-particle interactions to higher than normal temperatures (ambient ionospheric $T_{\mathrm{e}} / T_{\mathrm{i}}$ ratios are usually in the range of 1 to 2 ). A very preliminary analysis of the Differential Ion Flux Probe (DIFP) measure-

* In Stone et al. (1983), a value of 0.08 is given for the secondary-to-ram current ratio, as measured by the Differential Ion Flux Probe at a particular time. The ion current density in space can be calculated from the given value of collected instrument current by using the instrument characteristic, which includes angular effects on sensitivity. When this factor is included, the current density ratio is $\sim 0.3$ for the particular data in Stone et al. (1983). At other times, the ratio was found to be as high as $\sim 0.7$. 
ments indicates that the temperature of ions in the beam is in the range of a few hundred Kelvins. Although such low temperatures are abnormal for the ionospheric plasma, they may not be unreasonable for ions created within the Orbiter's neutral gas cloud. Outgasing products from the Orbiter would be expected to be in the range of $300 \mathrm{~K}$. The evidence of wave growth during the STS-3 mission also suggests that the ion beam temperature was much lower than the background ion temperature (Ashour-Abdalla and Okuda, 1986). Although a more detailed analysis is needed to verify the above results, we can assume that the beam ion temperature is less than, or equal to, the ambient ionospheric temperature. Although the source and mechanisms for the oblique ion streams are still under study, the possible generation of broadband noise has similarities to several existing one-dimensional models that were developed to explain the observation of electrostatic ion waves in the ionospheric and magnetotail regions (Kintner et al., 1981; Akimoto and Omidi, 1986; Grabbe and Eastman, 1984).

We have developed a two-dimensional theoretical model that describes the injection of an oblique ion beam into a background plasma and is directly applicable to the beam-plasma interaction that appears to have occurred in the environmental plasma of the Orbiter. While the model requires $T_{\mathrm{e}} / T_{\mathrm{i}} \gg 1$ for any significant wave growth to occur, as noted above, ratios as high as $T_{\mathrm{e}} / T_{\mathrm{i}}=6$ were observed at the Orbiter (Raith et al., 1984; Siskind et al., 1984). While the heating of the electrons probably results from wave-particle intcractions, we note that the ncutral gas cloud produced by the Orbiter is very extensive and apparently interacts with the ambient plasma; e.g. a significant disturbance of the ions was observed as far as $10 \mathrm{~m}$ upstream during the STS-3 mission (Stone et $a l ., 1986)$ and during the Spacelab-2 mission Orbiter outgasing products were observed several hundred meters upstream. It appears, therefore, that the neutral gas cloud is sufficiently extensive that if the oblique ion beams begin dumping free energy into the environment in the region of its upstream boundary, a sufficient interactive growth of broadband noise and electron heating could occur to explain the wave and particle observations prior to the Orbiter-mounted instruments passing through the region; i.e. the free energy of the beams would create electrostatic waves that would initially be Landau damped because $\mathrm{T}_{\mathrm{e}} \simeq \mathrm{T}_{\mathrm{i}}$ in the ambient ionosphere, but would, in this process, begin heating the electrons. As the electrons heat up, the instability created by the beam would begin to grow and a more efficient dumping of beam free-energy would occur and so on. The main objective of this paper is to show the feasibility of the predicted relationship between the broadband electrostatic noise and the secondary ion streams. This will be done by using the particle data obtained from the STS-3 mission in the theoretical model and comparing the characteristics of the predicted plasma instabilities and oscillations with the broadband noise observations from STS-3.

\section{THEORETICAL DERIVATION}

The physical situation under which the measurements of the Shuttle's particle and field environment in space were obtained is described in Stone et al. $(1983,1986)$ and Shawhan et al. (1984). In developing an appropriate model, we make a transformation from the Orbiter coordinate system to a new system in which the geomagnetic field is aligned with the $z$ axis and the relative drift velocity, $\mathbf{V}_{d}$, of the secondary ion stream (with respect to the ambient plasma) lies in the $y-z$ plane. The background electrons and ions are represented by local Maxwellian distribution functions, whereas the beam ions are represented by a drifting Maxwellian distribution function (Fig. 1). The wave frequency in this study is much higher than the ion cyclotron frequency but smaller than the electron cyclotron frequency, therefore it is reasonable to assume that the background electrons are magnetized while the background and stream ions are nonmagnetized. We also assume quasi-neutrality. Therefore, the total number density of background and stream ions is equal to the electron number density.

The derived generalized linear dispersion relation is essentially similar to several previous papers (for example, Grabbe and Eastman, 1984; Hudson and Roth, 1984; Omidi, 1985); this equation was obtained by using the usual method of integration along unperturbed trajectories. For perturbations of the type $\exp (\mathbf{i k} \cdot \mathbf{r}-\mathrm{iwt})$, the collisionless VlasovMaxwell equations yield the following dispersion relation:

$$
\begin{aligned}
& D(k, w)=1+\frac{1}{k^{2} \lambda_{\mathrm{e}}^{2}}\left\{1+\sum_{n} \Gamma_{n}\left(k_{\perp}^{2} \rho_{\mathrm{c}}^{2}\right) \frac{\omega}{k_{z} V_{T \mathrm{e}}}\right. \\
&\left.\times Z\left(\frac{\omega-n \Omega_{\mathrm{e}}}{k_{z} V_{T_{\mathrm{e}}}}\right)\right\}+\frac{1}{k^{2} \lambda_{\mathrm{i}}^{2}}\left\{1+\frac{\omega}{k V_{T \mathrm{t}}} Z\left(\frac{\omega}{k V_{T \mathrm{l}}}\right)\right\} \\
&+\frac{1}{k^{2} \lambda_{\mathrm{b}}^{2}}\left\{1+\frac{\left(\omega-\mathbf{k} \cdot \mathbf{V}_{\mathrm{d}}\right)}{k V_{T \mathrm{~b}}} Z\left(\frac{\omega-\mathbf{k} \cdot \mathbf{V}_{\mathrm{d}}}{k V_{T \mathrm{~b}}}\right)\right\}
\end{aligned}
$$

where $D(k, w)=D_{\mathrm{r}}(k, w)+\mathrm{i} D_{\mathrm{i}}(k, w), \lambda_{j}=V_{T j} / \sqrt{ } 2 w_{\mathrm{p} j}$ is the $j$ th species' Debye length, $w_{\mathrm{p} j}=\left(4 \pi N_{j} e^{2} / M_{j}\right)^{1 / 2}$ 


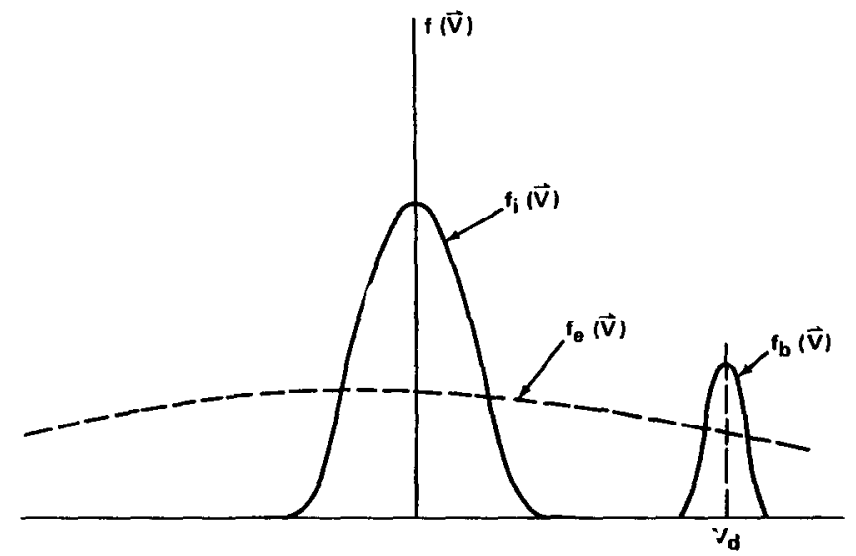

Fig. 1. THE SECONDARY ION STREAM VELOCITY DISTRIBUTION FUNCTION IN THE AMBIENT CHARGED PARTICLES' REFERENCE FRAME.

is the plasma frequency, $\rho_{j}=V_{T j} / \Omega_{j}$ is species' gyroradius, $\Omega_{j}=e B / M_{j} C$ is the cyclotron frequency of the $j$ th constituent, $\Gamma_{q}\left(v_{j}\right)=\mathrm{e}^{-v_{i}} I_{q}\left(v_{j}\right), I_{q}\left(v_{j}\right)$ is the modified Bessel function of order $q$, and $Z(\eta)=$ $Z_{\mathrm{r}}(\eta)+i Z_{\mathrm{i}}(\eta)$ is the plasma dispersion function (Fried and Conte, 1961). The wave frequency, $w$, is complex (i.e. $w=w_{\mathrm{r}}+\mathrm{i} w_{\mathrm{i}}$ ) with a positive growth rate. $\mathrm{By}$ assuming that $w_{\mathrm{i}} \ll w_{\mathrm{r}}$, equation (1) yields the linear frequency $w_{\mathrm{r}}$ and growth rate $w_{\mathrm{i}}$, where $w_{\mathrm{r}}$ satisfies $D_{\mathrm{r}}\left(k, w_{\mathrm{r}}\right)=0$ and the wave growth rate is given by:

$$
w_{\mathrm{i}}=\frac{-D_{\mathrm{i}}\left(k, w_{\mathrm{r}}\right)}{\frac{\partial D_{\mathrm{r}}\left(k, w_{\mathrm{r}}\right)}{\partial w_{\mathrm{r}}}} .
$$

\section{RESULTS AND DISCUSSIONS}

The properties of electrostatic ion waves can be determined by the propagation characteristics of the wave in the $w_{\mathrm{r}}-k$ plane under equation (1). Atomic oxygen ions $\left(\mathrm{O}^{+}\right)$are used as the ion species in this analysis, in accord with the observations from the Plasma Diagnostics Package (PDP) instruments (Grebowsky et al., 1983; Murphy et al., 1983). Since the frequency is measured by the moving wave instruments, the actual frequency is related to the measured frequency by $w^{\prime}=w-\mathbf{k} \cdot \mathbf{V}_{\mathrm{s}}$, where $V_{\mathrm{s}}$ is the shuttle velocity. The results from the model corresponding to the observed range of plasma conditions (i.e. $N_{\mathrm{e}}=3.0 \times 10^{6} \mathrm{~cm}^{-3}, \quad V_{\mathrm{d} z}=1.8 \times 10^{5} \mathrm{~cm} \mathrm{~s}^{-1}$ and $V_{\mathrm{d} y}=1.1 \times 10^{6} \mathrm{~cm} \mathrm{~s}^{-1}, T_{\mathrm{e}} / T_{\mathrm{i}}=2, T_{\mathrm{i}} / T_{\mathrm{b}}=7.5$ and $\left.N_{\mathrm{b}} / N_{\mathrm{c}}=0.5\right)$ are given in Fig. 2 . These results show that waves may be generated with frequencies ranging from $50 \mathrm{~Hz}$ up to several hundred kilohertz, and that the maximum growth rate occurs at frequencies in the range of $10 \mathrm{KHz}$, which is consistent with the observational data from $S T S-3$.

The angle, $\theta$, between the wave propagation vector and the ion beam is a parameter in these calculations and the results indicate that two types of wave modes may occur, i.e.the ion acoustic and the ion-ion modes. When $\theta<60^{\circ}$, the phase velocity is much greater than the background ion thermal velocity and the mechanism for wave generation is mainly due to the electron-ion beam interaction which produces ion acoustic waves. However, when $\theta>60^{\circ}$, the phase velocity is closer to the ion thermal velocity. In this case, a fluid type instability may occur (Tidman, 1967), and the wave may be produced by an instability resulting from the ion-ion interaction. The wave growth rate of this mode increases abruptly and reaches a maximum at $\theta \simeq 77^{\circ}$. As the ratio of $N_{\mathrm{b}} / N_{\mathrm{c}}$ is increased to 1, only the ion acoustic wave exists. However, as shown in Fig. 3, where $N_{\mathrm{b}} / N_{\mathrm{e}}=1$, the ion acoustic mode disappears when the wave propagation vector is nearly perpendicular to the ion beam $\left(\theta \geqslant 70^{\circ}\right)$. Since the ion-ion mode vanishes for $N_{\mathrm{b}} / N_{\mathrm{e}}=1$, Fig. 3 shows the characteristics of the pure ion acoustic wave mode.

Figure 4 shows the dependence of the maximum wave growth rate on the angle between the wave vector and the ion stream for several values of the temperature ratio, $T_{\mathrm{e}} / T_{\mathrm{i}}$. The plasma conditions are, otherwise, the same as those used for Fig. 2. When the $T_{\mathrm{e}} / T_{\mathrm{i}}$ ratio is small $(<1.3)$, the ion acoustic and ion-ion waves are restricted to very narrow areas at nearly parallel $\left(\theta=0^{\circ}\right)$ and nearly perpendicular $\left(\theta \simeq 76^{\circ}\right)$ directions, respectively (see Fig. 4). The 


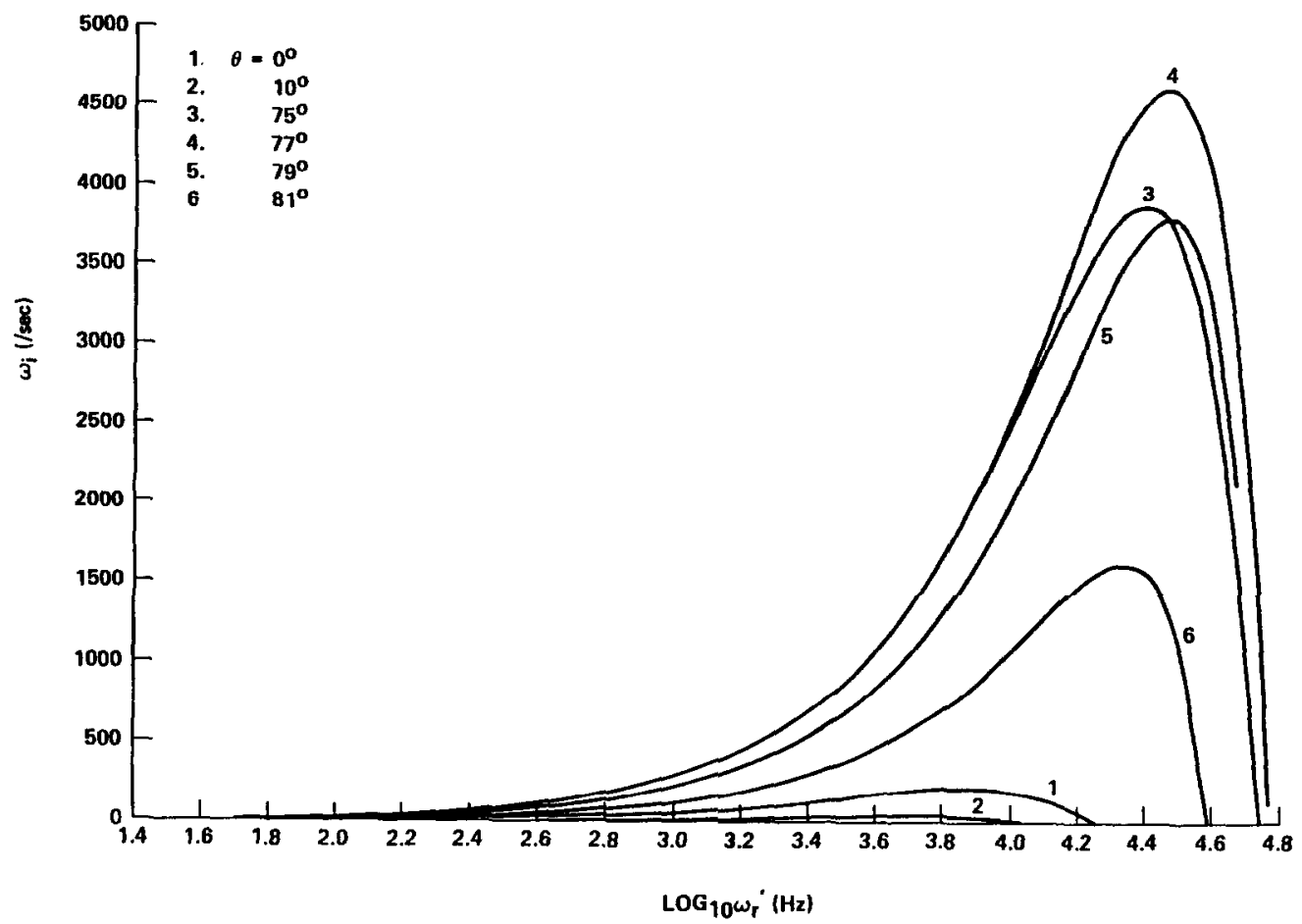

Fig. 2. The Growth Rate, $w_{\mathrm{j}}$, AS a Function of measured frequency, $w_{\mathrm{r}}^{\prime}$, AT $N_{\mathrm{e}}=3.0 \times 10^{6} \mathrm{~cm}^{-3}$, $V_{\mathrm{d} z}=1.8 \times 10^{5} \mathrm{~cm} \mathrm{~s}^{-1}, V_{\mathrm{d} y}=1.1 \times 10^{6} \mathrm{~cm} \mathrm{~s}^{-1}, T_{\mathrm{e}} / T_{\mathrm{i}}=2, T_{\mathrm{i}} / T_{\mathrm{b}}=7.5$ AND $N_{\mathrm{b}} / N_{\mathrm{c}}=0.5$, USING $\theta=\mathrm{cos}^{-1}$ $\left(\mathbf{k} \cdot \mathbf{V}_{\mathrm{d}}\right)$ AS A PARAMETER.

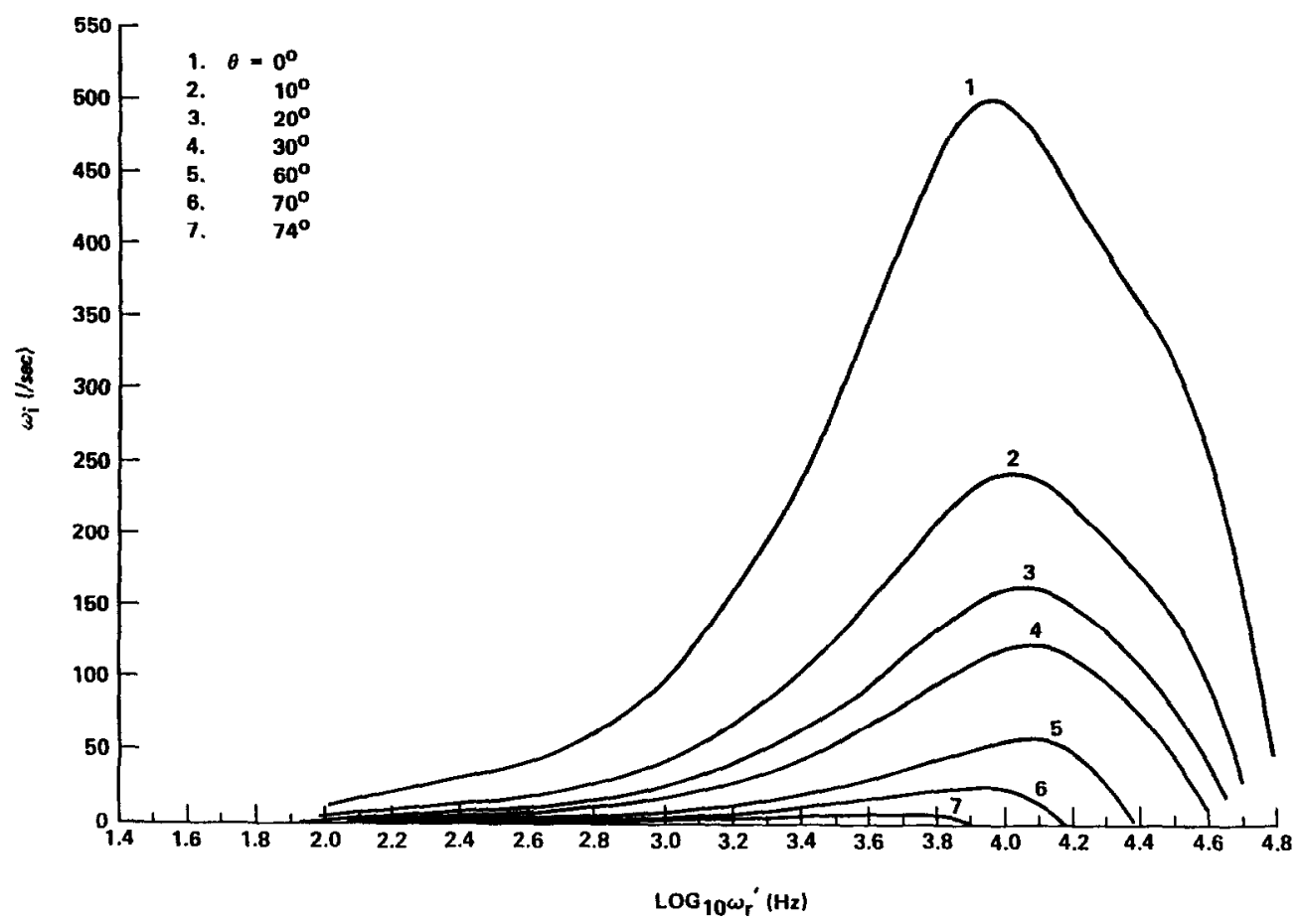

Fig. 3. THE GROWTH RATE, $w_{1}$, AS A FUNCTION OF MEASURED FREQUENCY, $w_{\mathrm{r}}^{\prime}$, FOR ELECTROSTATIC ION aCoustic WaVes at $N_{\mathrm{b}} / N_{\mathrm{e}}=1$, the Other Parameters are the SaMe as in Fig. 2. 


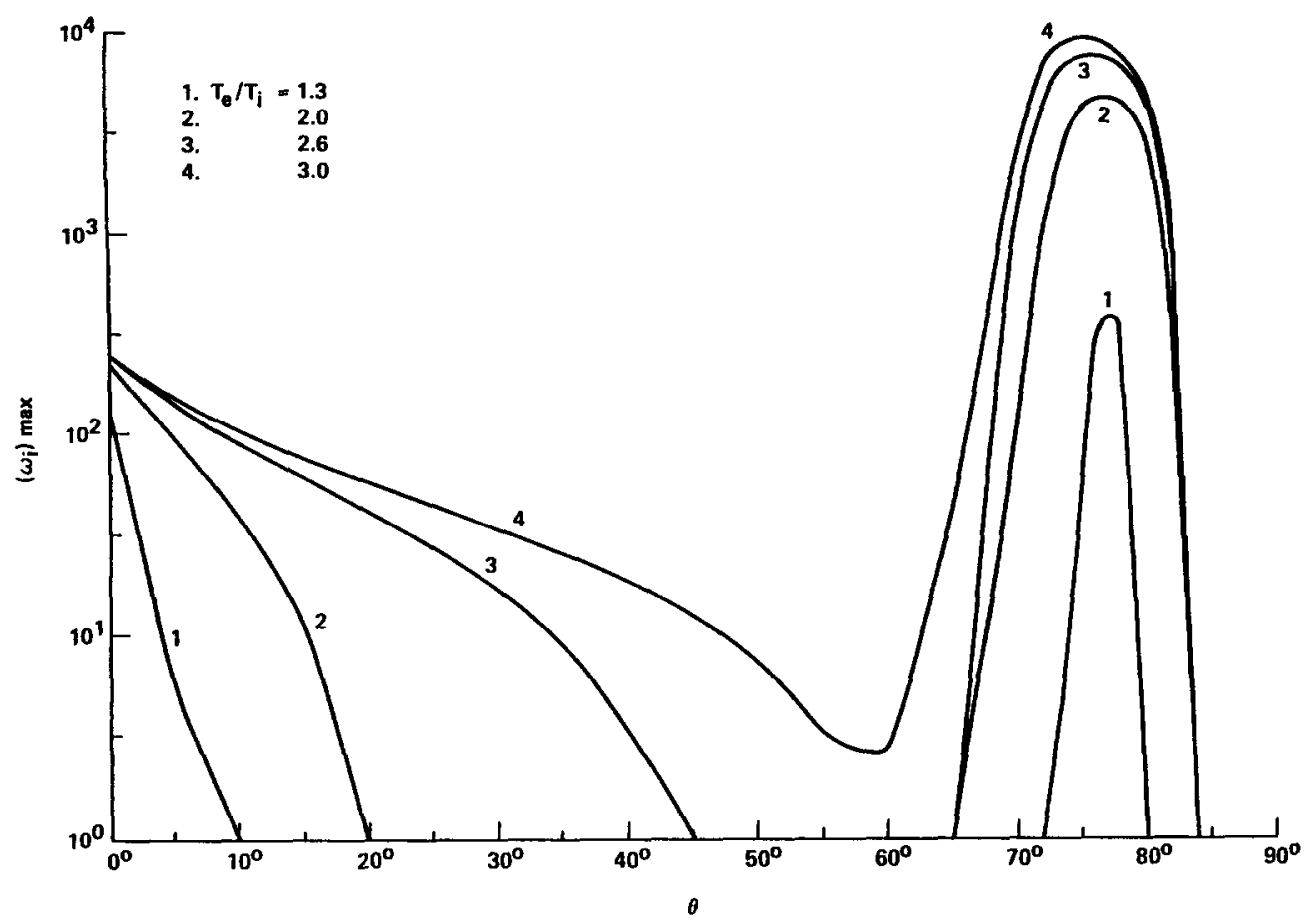

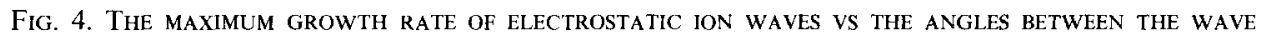
VECTOR AND THE ION STREAM, $\theta$, USING $T_{c} / T_{\mathrm{i}}$ AS A PARAMETER, THE OTHER PARAMETERS ARE THE SAME AS IN FIG. 2.

wave growth rate of both wave modes is small and comparable. As $T_{\mathrm{e}} / T_{\mathrm{i}}$ increases, the electron damping is gradually decreased and the interaction between the electrons and beam ions increases. Therefore, the wave growth rate for the ion acoustic mode increases and spreads over a wider angular range. When the $T_{\mathrm{e}} / T_{\mathrm{i}}$ ratio increases high enough such that the electron damping can be neglected, the ion acoustic wave begins to saturate in the parallel direction. At the same time, the ion-ion wave instabilities greatly enhance the wave amplitude in the nearly perpendicular direction. The results indicate that these two wave modes can only co-exist when the wave vectors of the two modes are nearly perpendicular. The ion-ion wave growth rate increases much faster than the ion acoustic wave mode as $T_{\mathrm{e}} / T_{\mathrm{i}}$ increases. This result indicates that the ion-ion instability heats the beam ions more than the ion acoustic instability. The higher the electron-ion tempertature ratio, the lower the instability threshold in the transition region. This is to be expected from the observed STS-3 thermal environment.

The maximum growth rate vs beam ion-electron number density ratio is presented in Fig. 5. The results show that for parallel propagation $\left(\theta=0^{\circ}\right)$, the wave growth rate monotonically increases with number density ratio for the ion acoustic mode. However, when the angle between the wave vector and the ion stream increases, the number density ratio at which the maximum growth rate occurs is between 0.3 and 0.8 , depending on the value of $\theta$. This is consistent with the results calculated by Omidi (1985). Again, we notice that the wave growth rate is much larger for the ion-ion wave mode than for the ion acoustic wave mode, except at higher $N_{\mathrm{b}} / N_{\mathrm{e}}$ ratios $(>0.9)$. The results also show that these two wave instabilities cannot grow immediately (see Fig. 5) at $N_{\mathrm{b}} / N_{\mathrm{c}}=0$ without a critical value of $N_{\mathrm{b}} / N_{\mathrm{e}}$ being reached, as Akimoto and Omidi (1986) suggested.

\section{CONCLUSIONS}

The Space Shuttle, with its environmental gas cloud, acts as a natural laboratory for the study of wave-particle interactions. The analysis and possible understanding of the interactions can be obtained through the use of in situ measurements coupled with theoretical analysis and numerial simulation. In this paper, we have studied the parametric behavior of the wave spectrum of broadband electrostatic noise in a magneto plasma with a superimposed drifting ion 


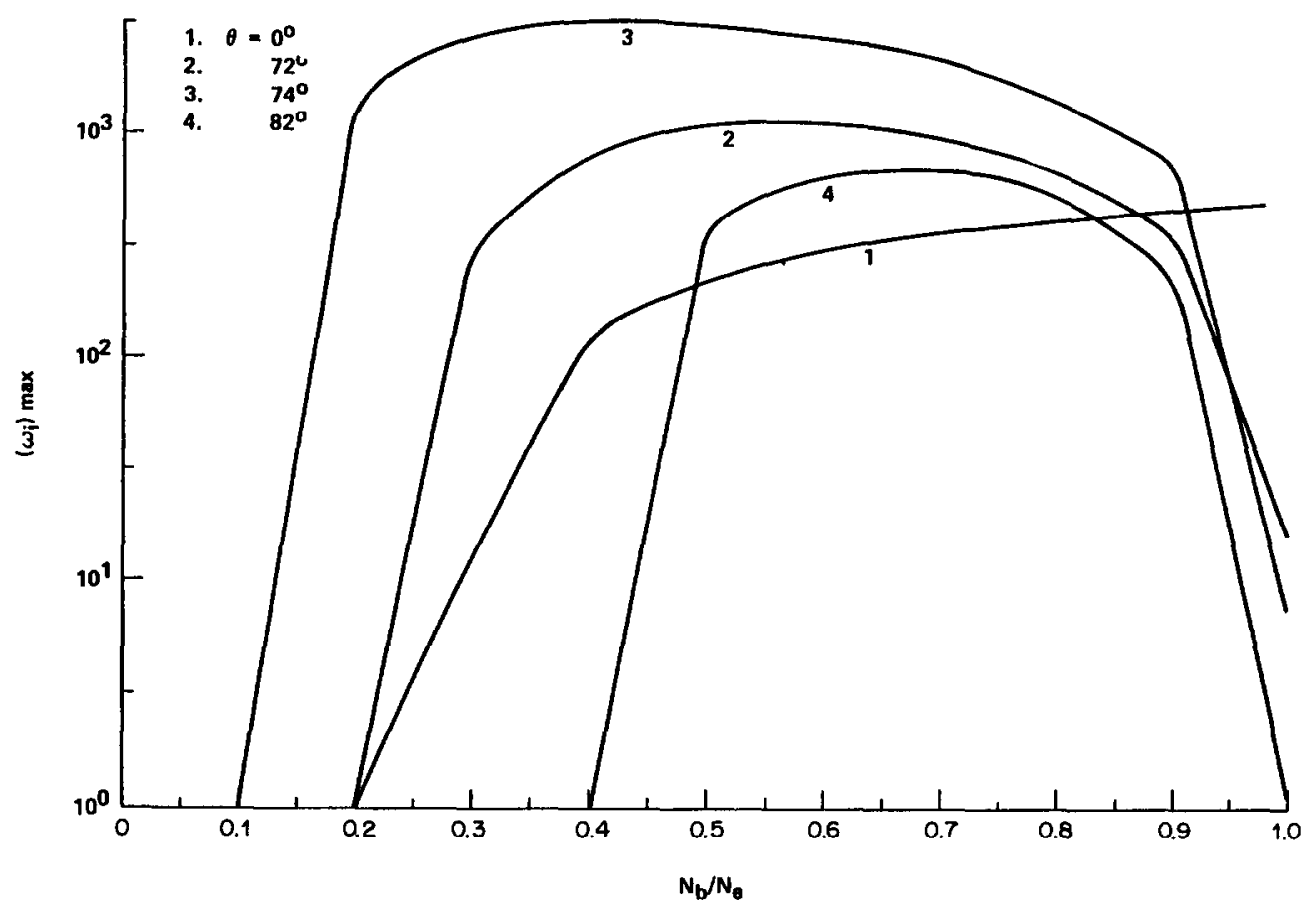

Fig. 5. The maximum growth rate vs $N_{\mathrm{b}} / N_{\mathrm{e}}$, USing $\theta$ as a Parameter, the other parameters are the SAME AS IN FIG. 2.

stream. The drifting ions are considered to be the source free energy for the waves, leading to the growth of an instability.

This linearized two-dimensional theory predicts the generation of waves over a broad band of frequencies, ranging from above the ion plasma frequency down to the ion cyclotron frequency, in agreement with the available in situ observations from the STS-3 mission. The instability derived in this manner produces ion acoustic wave and ion-ion wave emissions over a wide frequency range which corresponds to the observed frequency range for a reasonable choice of the plasma parameters. As we have shown earlier, the growth rate of the ion-ion wave mode is usually greater than the ion acoustic wave mode, except at higher ion beamelectron number density ratios or at lower electronion temperature ratios. Thus, in general, the ion-ion wave instability heats the beam ions more than the ion acoustic instability does.

During the IMP 8 mission, Gurnett et al. (1976) found that the broadband electrostatic noise was generated over most wave vector directions with the most intense waves occurring in the direction nearly perpendicular to an observed field-aligned current. The theoretical analysis by Omidi (1985) was shown to be consistent with the observations. The generation of electrostatic waves by the ion streams in the Orbiter's environmental plasma is similar in some aspects to wave-particle interactions observed in the Earth's geomagnetotail. This phenomena therefore provides an example of how processes that occur in natural space plasma phenomena, can be created and studied in more detail in the ionosphere. Although the measurements form the STS-3 mission are unable to specify the direction at which the maximum growth rate occurred, we do predict results for the STS-3 missions similar to those for IMP 8. During the Spacelab 2 mission, the PDP was spinning while in free flight and, therefore, the wave vector can be determined. The results of these measurements, when available, will provide a more viable test for the present model.

In conclusion, we note that simulation calculations by Ashour-Abdalla and Okuda (1986) predict electron heating to occur in a similar plasma model. Although, theoretically, the wave instability could heat the electron population (Davidson et al., 1970) which, in turn, could ionize the neutral gas cloud, the evaluation of the possible connection between the broadband noise, electron heating, and neutral particle ionization will re- 
quire a careful consideration of electric field strengths and the efficiency of coupling with the electrons as well as wave growth rates. The present study has concentrated on the generation of ion acoustic and ion-ion waves. The authors are aware that other important ion wave modes, i.e. the electrostatic ion cyclotron and ion Bernstein waves, have received no attention at this point in the analysis. According to the observations, waves can also occur with the maximum growth rate at a lower frequency near the $N$ th order of the ion cyclotron frequency range. A detailed study of this equally important wave mode will be presented elsewhere. However, the present model, although incomplete, is adequate to show that the free energy associated with the high inclination ion streams is sufficient to ignite ion acoustic and/or ion-ion wave instabilities that are capable of producing the observed broadband noise signature.

Acknowledgements - The authors would like to thank D Gurnett and G. Murphy of the University of Iowa for supplying the wave data from PDP/STS-3 measurements. We would also like to thank Wayne Thompson of Boeing Computer Support Services (now at Sparta, Inc.) for reduction of the DIFP data. K.S.H. acknowledges support from the National Research Council under their resident Research Associateship program. K.H.W. acknowledges support from NASA grant NAG8-058. U.S. acknowledges support from NASA grant NGR 23-005-320.

\section{REFERENCES}

Akimoto, K. and Omidi, N. (1986) The generation of broadband electrostatic noise by an ion beam in the magnetotail. Geophys. Res. Lett. 13, 97.

Ashour-Abdalla, M. and Okuda, H. (1986) Theory and simulations of broadband electrostatic noise in the geomagnetic tail. J. geophys. Res. 91, 6833.

Davidson, R., Krall, N., Papadopoulos, K. and Shanny, R. (1970) Electron heating by electron-ion beam instabilities. Phys. Res. Lett. 24, 579.

Fried, B. and Conte, S. (1961) The Plasma Dispersion Function. Academic Press, New York.

Grabbe, C. L. and Eastman, T. E. (1984) Generation of broadband electrostatic noise by ion beam instabilities in the magnetotail. J. geophys Res. 89, 3865.

Grebowsky, J. M., Pharo, M. M., III, Taylor, H. A. and Eberstein, 1. J. (1983) Measured thermal ion environment of STS-3. AIAA Shuttle Environment and Operations Meeting, 31 October-3 November 1983, Washington, DC. Paper No. AIAA-83-2597.

Gurnett, D., Frank, L. A. and Lepping, R. (1976) Plasma in the distant magnetotail. J. geophys Res. 81, 6059 .

Hudson, M. K. and Roth, I. (1984) Thermal fluctuations from an artificial ion beam injection into the ionosphere. J. geophys. Res. 89, 9812.

Kintner, P. M., Kelley, M. C., Holmgren, G. and Bostrom, R. (1981) The observation and production of ion acoustic waves during the Trigger experiment. J. geophys Res. 85, 5071 .

Murphy, G. B., Shawhan, S. D., Frank, L. A., D’Angelo, N., Gurnett, D. A., Grebowsky, J. M., Reasoner, D. L. and Stone, N. H. (1983) Interactions of the Space Shuttle Orbiter with the ionospheric plasma. Proc. 17th ESLAB Symp. on Spacecraft Plasma Interactions and their Influence on Field and Particle Measurements, Noordwijk, The Netherlands. Eur. Space Agency, ESA SP-198, pp. 73-78.

Omidi, N. (1985) Broadband electrostatic noise produced by ion beams in the Earth's magnetotail. J. geophys. Res. 90, 12330.

Raitt, W. J., Siskind, D. E., Banks, P. M. and Williamson, P. R. (1984) Measurements of the thermal plasma environment of the Space Shuttle. Planet. Space Sci. 32, 457.

Scarf, F., Frank, L., Ackerson, K. and Lepping, R. (1974) Plasma wave turbulence at distant crossings of the plasma sheet boundaries and netural sheet. Geoophys Res. Lett. $1,189$.

Shawhan, S. D., Murphy, G. B. and Picket, J. S. (1984) Plasma Diagnostics Package assessments of the STS-3 Orbiter plasma environment. J. Spacecraft Rockets 21, 387.

Siskind, D. E., Raitt, W. J., Banks, P. M. and Williamson, P. R. (1984) Interactions between the orbiting Space Shuttle and the ionosphere. Planet. Space Sci. 32, 881.

Stone, N. H., Samir, U., Wright, K. H., Jr., Reasoner, D. L. and Shawhan, S. D. (1983) Multiple ion streams in the near vicinity of the Space Shuttle. Geophys. Res. I.ett. 10, 125.

Stone, N. H., Wright, K. H., Jr., Hwang, K. S., Samir, U., Murphy, G. B. and Shawhan, S. D. (1986) Further observations of space shuttle plasma-electrodynamic effects from $O S S-1 / S T S-3$. Geophys. Res. Lett. 13, 217.

Tidman, D. A. (1967) Turbulent shock waves in plasma. Physics Fluids 10, 547. 